\title{
CORRIGENDUM
}

\section{Lifestyle factors are determinants of children's blood pressure levels: the CYKIDS study}

\author{
C Lazarou, DB Panagiotakos and A-L Matalas
}

Journal of Human Hypertension (2011) 25, 752. doi:10.1038/jhh.2011.84

Correction to: The Journal of Human Hypertension (2009) 23, 456-463; doi:10.1038/jhh.2008.151; published online 18 December 2008

Since the publication of the above paper, the authors have noticed an error in the abstract section.

The sentence 'Finally, compared with those with at least an average KIDMED score, children with low score were $75 \%$ less likely to have elevated DBP levels, whereas they exhibited a nonsignificant trend for lower SBP (by 29\%) and lower overall BP levels (by 30\%).' is incorrect and should read as follows:

'Finally, compared with those with low KIDMED score, children with at least an average score were $75 \%$ less likely to have elevated DBP levels, whereas they exhibited a nonsignificant trend for lower SBP (by 29\%) and lower overall BP levels (by 30\%).'

The authors would like to apologize for this mistake. 